\title{
Application of SAFY Crop Growth Model for Maize Yield Forecast
}

\author{
Igor I. Sereda, Olga V. Tutubalina \\ Lomonosov Moscow State University, Moscow, Russia \\ iisereda@mail.ru
}

\begin{abstract}
Usually, yield prediction requires a significant number of parameters, which are currently impossible to obtain using just remote sensing data. Using the SAFY model, we developed a method of above-ground phytomass estimation and yield forecast on the basis of meteorological information, Sentinel 2 MSI imagery and literature data. We tested the method for an experimental maize field in the Lipetsk region of Russia. As a result of our study, we estimated the total above-ground phytomass and yield one and a half months before harvest for the field. The maximum error in determining the yield using the SAFY crop development model, can be tentatively estimated at $\pm 10 \%$.
\end{abstract}

Keywords: precision agriculture, maize, yield, above-ground phytomass, crop growth model, SAFY 


\title{
ИСПОЛЬЗОВАНИЕ МОДЕЛИ РАЗВИТИЯ РАСТЕНИЙ SАFY ДЛЯ ПРОГНОЗИРОВАНИЯ УРОЖАЙНОСТИ НА ПРИМЕРЕ ПОСЕВОВ КУКУРУЗЫ
}

\author{
И.И. Середа, О.В. Тутубалина \\ Московский государственный университет имени М.В. Ломоносова, Москва, Россия \\ iisereda@mail.ru
}

\begin{abstract}
Как правило, для прогноза урожайности требуется значительное количество параметров, которые в настоящее время невозможно получить, используя исключительно данные дистанционного зондирования. С помощью модели SAFY мы разработали метод определения зеленой фитомассы и прогнозирования урожайности на основе метеорологической информации, данных съемочной системы Sentinel-2 MSI и литературных источниках. Мы проверили этот метод на экспериментальном кукурузном поле в Липецкой области (Россия). В ходе исследований, мы рассчитали общий объем зеленой фитомассы и урожайность для этого поля за полтора месяца до уборки урожая. Максимальная погрешность определения урожайности с помощью модели развития культур SAFY может быть приблизительно оценена как \pm 10 \%.
\end{abstract}

Ключевые слова: дистанционное зондирование, точное земледелие, кукуруза, урожайность, зеленая фитомасса, модель развития растений, SAFY.

\section{Введение}

Для обеспечения оптимальной урожайности уже долгое время исследуется взаимосвязь между ростом сельскохозяйственных культур и условиями окружающей среды. При этом создавались модели, позволяющие оценить их состояние и спрогнозировать развитие. После почти 40 лет развития, модели прогнозирования урожайности продвинулись от первоначального, качественного, до количественного моделирования роста сельскохозяйственных культур [1].

Модели развития сельскохозяйственных культур изначально показывали развитие посевов на полях с хорошо известными и равномерно распределенными почвенными, климатическими и агрономическими характеристиками. Поэтому возникают некоторые проблемы при применении этих моделей для исследования больших площадей, так как на них перечисленные показатели сильно изменяются как в пространственном, так и во временном отношении. Следовательно, применение данных дистанционного зондирования в расчете моделей развития растений является необходимым, позволяя учитывать фактор пространственной неоднородности.

Цель данной работы - исследовать возможность определения фитомассы и прогнозирования урожайности зерновой кукурузы по данным съемочной системы Sentinel-2 MSI и модели развития растений на примере экспериментального кукурузного поля в Липецкой области при минимально возможном объеме полевых данных.

Использование наиболее распространенных и хорошо описанных на данный момент групп моделей развития растений, таких как WOFOST, AquaCrop и AGROTOOL [2; 3; 4], сопряжено с некоторыми трудностями. Для их корректной работы необходимо учитывать такие 
факторы, как радиационный режим посева, турбулентный режим воздухо- и влагообмена в верхних слоях почвы, распределение накопленных продуктов фотосинтеза по органам растения, транспирация растений, динамика соединений азота в почве. Поэтому применение этих моделей обосновано только при наличии перечисленных параметров.

Сбор таких подробных данных на значительных площадях сопряжен с необходимостью использования значительного количества материальных и трудовых ресурсов. В связи с тем, что подобными данными об экспериментальном поле мы не располагали, было решено применить более простую модель развития растений SAFY (Simple algorithm for yield estimates), созданную на основе базового уравнения для первичной чистой продукции [5]. Эта модель привлекательна тем, что осуществляет прогноз урожайности посевов при наличии минимальной полевой информации (фенологическое состояние культур, температура и приходящая солнечная радиация).

\section{Методика}

SAFY относится к моделям развития культур, которые сочетают в себе использование базовых характеристик растительности и данных дистанционного зондирования. Согласно проведенным исследованиям [6; 7], она позволяет спрогнозировать урожайность при наличии метеоданных, справочной информации и достаточной серии космических снимков (хотя бы один снимок для каждой фенологической стадии развития посевов).

Применение модели SAFY позволяет вычислять изменения индекса зеленой листовой поверхности (Green Leaves Area Index: GLAI), наземной сухой фитомассы (Dry Aerial Matter: DAM) и непосредственно урожая (Grain Yield: GY). Для работы модели необходимы ежедневные вычисления прироста фитомассы, которые выполняются на основе уравнения Дж.Л. Монтейта [5]. Это уравнение связывает количество прироста фитомассы (в пересчете на сухой вес) с фотосинтетически активной радиацией (Portion of Solar Radiation: PAR), поглощенной растениями.

Ежедневный прирост фитомассы зависит от таких факторов, как:

1. Суммарная солнечная радиация (incoming global radiation: $\mathrm{R}_{\mathrm{g}}$ );

2. Климатическая эффективность (climatic efficiency: $\varepsilon_{C}$ ), которая является

отношением приходящей фотосинтетически активной радиации к суммарной солнечной радиации;

3. Фракция поглощенной фотосинтетически активной радиации, или эффективность перехвата света (FAPAR) - доля фотосинтетически активной радиации, поглощенной растениями.

4. Эффективность использования света (effective light-use efficiency: ELUE), которое является отношением фитомассы к энергии, затраченной на ее производство.

5. Функция температуры $\mathrm{F}_{\mathrm{t}}\left(\mathrm{T}_{\mathrm{a}}\right)$, которая корректирует оценку ежедневного прироста фитомассы в зависимости от того, насколько средняя температура воздуха в этот день была оптимальной для роста культуры.

Таким образом, итоговое уравнение Монтейта, показывающее ежедневный прирост фитомассы ( $\triangle \mathrm{DAM})$, выглядит следующим образом:

$$
{ }_{\triangle} \mathrm{DAM}=\mathrm{R}_{\mathrm{g}} \times \varepsilon_{\mathrm{c}} \times \mathrm{FAPAR} \times \mathrm{ELUE} \times \mathrm{F}_{\mathrm{T}}\left(\mathrm{T}_{\mathrm{a}}\right)
$$

Так как поглощенная фотосинтетически активная радиация (Absorbed Photosynthetically Active Radiation: APAR) является произведением фотосинтетически активной радиации и эффективности перехвата света, в работе Дучемина [6], это уравнение было преобразовано в следующий вид:

$$
{ }_{\triangle} D A M=A P A R \times E L U E \times F_{T}\left(T_{a}\right)
$$

Для того чтобы узнать общий вес фитомассы, произведенной на некоторой площади в течение сезона (DAMtot), необходимо сложить весь ее ежедневный прирост. 


$$
D A M_{\text {sot }}=\sum_{x}^{y} \Delta D A M
$$

где х и у - первый и последний дни, в которых проводились измерения.

Для того, чтобы вычислить собранный урожай, используется индекс урожайности НІ (Harvest Index), который равен отношению массы собранного зерна или полезных частей растений к их общей фитомассе. Таким образом, урожай (GY) будет равен:

$$
G Y=H I \times D A M_{\text {tot }}
$$

Индекс урожайности варьируется в зависимости от культуры. Для кукурузы он равен 0.52 [8]. Таким образом, для того, чтобы вычислить фитомассу растений, а также урожайность посевов, необходимо определить поглощенную фотосинтетически активную радиацию (APAR), эффективность использования света и функцию температуры.

Для расчета значений фракции поглощенной фотосинтетически активной радиации (FAPAR) были использованы данные снимков Sentinel-2 MSI на территорию экспериментального поля в Липецкой области. На этот участок удалось найти и обработать 5 безоблачных снимков Sentinel-2 MSI (таблица 1).

Таблица 1. Снимки Sentinel-2 MSI на территорию экспериментального поля в Липецкой области и рассчитанные по ним значения FAPAR

\begin{tabular}{|c|c|c|c|}
\hline Дата & День года & $\begin{array}{c}\text { Дней от предыдущего } \\
\text { снимка }\end{array}$ & FAPAR \\
\hline 01.05 .2016 & 121 & 0 & 0 \\
\hline 18.06 .2016 & 170 & 49 & 0,35 \\
\hline 21.06 .2016 & 173 & 3 & 0,51 \\
\hline 02.07 .2016 & 184 & 11 & 0,65 \\
\hline 07.08 .2016 & 220 & 36 & 0,71 \\
\hline 28.08 .2016 & 241 & 21 & 0,62 \\
\hline
\end{tabular}

B программном обеспечении SNAP, разработанном Европейским космическим агентством (ESA), благодаря модулю Biophysical Processor возможно рассчитать значения FAPAR. При этом в качестве алгоритма используется нейронная сеть, которая имеет 11 входных параметров: значения КСЯ в каналах В3, B4, B5, B6, B7, B8a, В11 и В12, а также косинусы зенитного угла съемки, зенитного угла Солнца и относительного азимутального угла (viewing zenith, sun zenith, relative azimuth angle). Расчет значений FAPAR ведется на основе базы эталонов этой нейронной сети, полученной по полевым данным. Согласно моделям, представленным в работах Р. Минени и А. Руими [9; 10], ELUE может быть определено как фиксированное значение для конкретного типа растительности, имеющее относительно стабильное изменение в течение сезона. В связи с этим, значения ELUE были взяты на основе работы А.А. Гительсона и Дж.А. Гамона [11]. В ней для экспериментальных посевов кукурузы, расположенных в 58 км севернее города Линкольн (Небраска, США), с 2001 по 2008 гг рассчитывались значения эффективности поглощения света в течение сезона. Эти значения были использованы в модели для Липецкой области, так как являлись наиболее достоверными и приближенными по климатическим условиям из найденных в научной литературе.

Функция оптимальной температуры рассчитывалась на основе отклонений фактических значений температуры от оптимальных для кукурузы в определенные стадии ее сезонного развития. В результате расчетов она принимает значения от 0 до 1 для каждого из дней наблюдений.

Полевыми данными, которые были использованы в расчете модели SAFY, являлись только сроки сева, даты наступления фенологических фаз развития культуры и фактическое значение урожайности в пересчете на стандартную влажность. Эту информацию нам 
предоставила компания ООО “Сингента”, которой принадлежало экспериментальное поле. Метеорологические данные (температура, приходящая солнечная радиация, облачность) были получены на основе архивных метеорологических данных.

Особенностью работы модели SAFY, благодаря которой становится возможным прогноз урожайности с ее использованием, является тот факт, что параметр зеленой фитомассы растений достигает максимума для посевов кукурузы за 1-1,5 месяца до фактического сбора урожая. То есть, приостанавливается развитие вегетативных частей растений и происходит дальнейший налив зерна. Поскольку модель SAFY позволяет оценить зависимость между значениями зеленой фитомассы и фактической урожайностью, то прогноз урожая становится возможным выполнить заранее (Рис. 1).

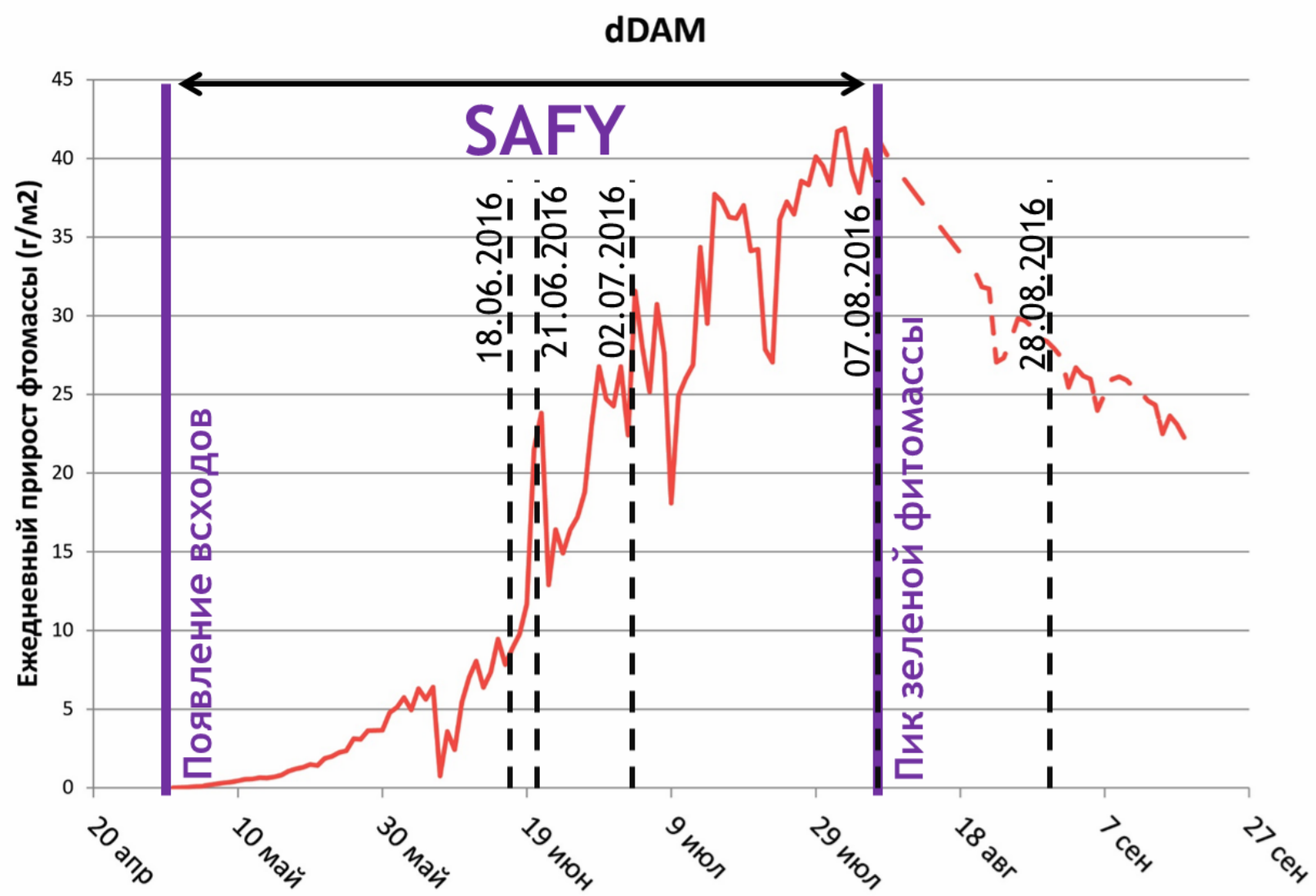

Рис. 1. График ежедневного прироста фитомассы (г/м²). Для расчета общей фитомассы и итоговой урожсайности используется период наблюдений с момента появления всходов до наступления пиковых значений зеленой фитомассы. Пунктирными линиями показаны даты съемок спутниковой системы Sentinel-2 MSI

\section{Результаты}

В результате вычислений, проведенных на основе модели SAFY, итоговая расчетная фитомасса (на дату максимума зеленой фитомассы) для посевов кукурузы на экспериментальном поле в Липецкой области составила $1,84 \mathrm{\kappa} / \mathrm{m}^{2}$, а итоговая расчетная урожайность - 96,2 ц/га. При этом фактическое значение урожайности в пересчете на стандартную влажность, составило 94,5 ц/га. Таким образом, модельные результаты близки к фактическим (разница 1,7 ц/га).

Важно упомянуть, что при расчете SAFY использовались исходные данные, которые имеют ту или иную степень погрешности. Итоговые значения урожайности могут меняться в зависимости от каждого из параметров. 
Существенное влияние оказывает изменение такого параметра, как даты стадий созревания кукурузы, так как для каждой стадии характерны свои оптимальные температуры. При смещении временных рамок стадий созревания культуры (которые определялись исходя из доступных космических съемок) на 10 дней, итоговые значений урожайности изменяются на 5-6 ц/га. Точное знание начала необходимых стадий созревания может минимизировать данную ошибку. Возможно, также, что каждого отдельного гибрида культуры оптимальные температуры могут отличаться, но это не было оценено, поскольку гибриды кукурузы занимали слишком узкие полосы по сравнению с разрешением космических снимков.

Согласно документации инструмента Biophysic processor программы SNAP, максимальная погрешность определения FAPAR составляет 0.1 , или $10 \%$ [12]. Так как обычно суммарную ошибку, складывающуюся из нескольких, считают как корень из суммы квадратов ошибок [13], то на основе четырех измерений итоговые значения урожайности изменятся на 8 ц/га.

Различия между предсказанными и фактическими значениями ELUE также вводит погрешность. Однако она не так высока. При изменении всех значений этого параметра на 0,2 (приблизительно на 10\% для максимальных значений и около 50\% для минимальных значений параметра), итоговая урожайность изменится на 1-2 ц/га.

B расчете модели SAFY в качестве коэффициента климатической эффективности использовалась глобальная постоянная 0,48. Однако она может отличаться от фактических ее значений для территории Липецкой области. Кроме того, модель не учитывает негативные факторы воздействия на зерно на стадии его созревания после достижения максимальной зеленой фитомассы.

Таким образом, погрешность определения урожайности с помощью модели развития культур SAFY можно примерно оценить как \pm 10 ц/га. Однако вероятность максимальной ошибки при этом достаточно низкая. Основную долю ошибки вносит погрешность при определении FAPAR. Из этого следует вывод, что для улучшения работы модели необходимо получить как можно больше значений FAPAR по разновременным снимкам Sentinel-2 MSI, a также точно определить стадии созревания растительности.

\section{Выводы}

Среди положительных особенностей использования модели SAFY можно отметить, что она способна достаточно эффективно определять значения фитомассы и урожайности посевов кукурузы без учета статистических данных предыдущих лет. При этом Становится возможным прогнозирование этих параметров за 1-1,5 месяца до фактического сбора урожая.

Тем не менее, для ее применения все еще необходимо определять значительное число переменных (не менее 11), каждая из которых имеет свою погрешность. Работоспособность модели также сильно зависит от точности определения дня достижения максимальной зеленой фитомассы. Для определения этой даты целесообразно определять максимальные сезонные значения вегетационного индекса NDVI с использованием космических съемочных систем с высоким временным разрешением (таким, как MODIS). Кроме того, модель SAFY не способна учесть последствия стрессовых ситуаций, которые происходили на поле после наступления максимальных значений зеленой фитомассы (засуха, болезни, и т.д.).

В настоящий момент ведутся работы по тестированию описанной методики для других посевов и территорий, так как фактическую точность модели нельзя оценивать по результатам одного исследования. Тем не менее, использование модели SAFY на примере экспериментального поля в Липецкой области позволяет сделать вывод, что она работоспособна и показывает правдоподобный результат. 


\section{References}

[1] Jin X., Kumar L., Li Zhenhai, Feng H., Xu Xingang, Yang G., Wang J., A rewiew of data assimilation of remote sensing and crop models, European Journal of Agronomy, 2018, 92, pp. 141-152.

DOI: https://doi.org/10.1016/j.eja.2017.11.002

[2] Poluektov R.A., Terleev V.V., Imitacionno-modeliruyushchij kompleks AGROTOOL, v.3 (Dinamicheskaya model' produkcionnogo processa sel'skohozyajstvennyh rastenij): Algoritmicheskaya struktura modeli. Rossijskaya akademiya sel'skohozyajstvennyh nauk. Agrofizicheskij nauchno-issledovatel'skij institut. Laboratoriya modelirovaniya agroekosistem, St.P., 2007, pp. 95-112. (In Russian)

[3] Diepen C.A. van, Rappold C., Wolf J, CWFS Crop Growth Simulation Model WOFOST Documentation: Version 4.1. Centre for World Food Studies, Wageningen, The Netherlands, 1988, pp. 1-299.

[4] Steduto P., Hsiao T.C., Raes D., Fereres E., AquaCrop-the FAO crop model to simulate yield response to water. I. Concepts and underlying principles. Agron, 2009, pp. 426-437. DOI: 10.2134/agronj2008.0139s

[5] Monteith J. L., Climate and efficiency of crop production in Britain. Philosophical Transactions of the Royal Society of London. Series B, Biological Sciences. 1977, 281, pp. 277-294.

[6] Duchemin B., Maisongrande P., Boulet G., Benhadj I. A simple algorithm for yield estimates: Evaluation for semi-arid irrigated winter wheat monitored with green leaf area index, Environmental Modeling \& Software, 2008, 23, pp. 876-892. DOI: 0.1016/j.envsoft.2007.10.003.

[7] Claverie M., Demarez V., Duchemin B., Hagolle O., Ducrot D., Marais-Sicre C., Dejoux J.-F., Hux M., Keravec P., Beziat P., Fieuzal R., Ceshia E., Dedieu G. Maize and sunflower biomass estimation in southwest France using spatial and temporal resolution remote sensing data, Remote Sensing of Environment, 2012, 24, pp. 844-857. DOI: $10.1016 /$ j.rse.2012.04.005.

[8] Atwell B.J., Kriedemann P.E., Turnbull C.G.N., Plants in action: Adaptation in Nature, Performance in Cultivation, Macmillan Education Australia Pty Ltd, Melbourne. Australia. 1999.

DOI: https://doi.org/10.1006/anbo

[9] Myneni R.B., Los S.O., Asrar G., Potential gross primary productivity of terrestrial vegetation from 19821990, Geophysical Research Letters, 1995, 22, pp. 2617-2620. DOI: https://doi.org/10.1029/95GL02562

[10] Ruimy A., Saugier B., Dedieu G. Methodology for the estimation of terrestrial primary production from remotely sensed data. Journal of Geophysical Research, 1994, 99, pp. 5263-5283. DOI: 10.1029/93JD03221

[11] Gitelson A.A., Gamon J.A., The need for a common basis for defining light-use efficiency: Implications for productivity estimation, Remote Sensing of Environment, 2015, 156, pp.196-201. DOI: 10.1016/j.rse.2014.09.017

[12] Weiss M. S2ToolBox Level 2 products: LAI, FAPAR, FCOVER, Sentinel2 ToolBox Level2 Products, Date Issued 02.05.2016. Issue:V1.1, 53 p.

[13] Andreev A.D., Chernyh L.M. Fizika. Obrabotka rezul'tatov izmerenij v fizicheskom praktikume: Konspekt lekcii, St.P GUT, 2009. 8 p. (In Russian). 